\title{
Nanoparticles Size Distribution Assessment During Early Synthesis Stages
}

\author{
Dan Chicea ${ }^{1, *}$, Mirela Maria Codescu2 ${ }^{2}$ Alexandra Nicolae ${ }^{1}$, Oleksandr Doroshkevych ${ }^{3}$, \\ Akhmed Islamov ${ }^{3}$, and Miroslaw Kulik ${ }^{3}$ \\ ${ }^{1}$ Faculty of Sciences, Lucian Blaga University of Sibiu, Dr. Ion Raţiu Street 5-7, Sibiu, Romania \\ ${ }^{2}$ INCDIE ICPE-CA Bucharest, Romania \\ ${ }^{3}$ Frank Laboratory of Neutron Physics (FLNP), Joint Institute for Nuclear Research, Dubna, Russia
}

\begin{abstract}
Nanotechnology, an innovative field of material science, designs and produces nanostructures materials with unique properties that make them suited for various bioengineering applications. The shape and the dimension are the main variables depending on the method of synthesis or chemical precursors. Silver nanoparticles obtained through chemical synthesis exhibit as strong antibacterial effect if their size lays in a well-defined range. However, a small size of such particles, in the range of nanometers to several tens of nanometers, requires specific techniques as Dynamic Light Scattering or Transmission Electron Microscopy, both being affected by certain assumptions or by the high cost and long time required for a measurement. A nonconventional method based on Direct Particle Tracking can be used to produce the particle size distribution in the early nanoparticle synthesis stages. The work presents a realistic computer simulation of nanoparticle diffusion performed with the CHODIN code and a size distribution assessment using a code for Direct Particle Tracking written for this purpose. The results are consistent with the particle size used for simulation and states as a proof of concept for using the Direct Particle Tracking method for particle size distribution in the early stages of nanoparticle synthesis. Keywords: Nanoparticles, Size distribution, Diffusion Simulation, Direct Particle Tracking
\end{abstract}

\section{Introduction}

Nanotechnology, the science of low-dimensional structures, is categorized as a branch of multidisciplinary sciences, such as physics or chemistry, studied at atomic and molecular level. The phenomena studied and the nanoscale experiments performed by researchers over years have the purpose to change the manufacturing process of many devices and to improve the level of understanding in material's structure [1].

Nanotechnology is divided by different criteria in many categories [2, 3]. The main important aspect remains the small dimension of materials, therefore the dimension of materials and the effects of this property on internal structure is considered the most redundant property. Nanoparticles, materials with dimensions below 100 nanometers, are an interesting subject due to their unique properties [4]. The ability of having an individual pattern, comparing to a bulk

\footnotetext{
*Corresponding author: dan.chicea@ulbsibiu.ro
} 
size, have proved to offer efficiency in many experiments. Their most important property, a large surface-area volume, allows their use in many applications such as conductors, catalyzers, transport vesicles or even as drug carrier for very precisely utilization.

Metal nanoparticles gained a high attention due to their anti-microbial and optical properties. Silver nanoparticles, along with zirconia, zinc oxide or titanium dioxide for example, possess anti-bacterial properties for many types of microorganisms. Silver nanoparticles are extremely versatile regarding their characteristics [5]. These metal-oxide nanoparticles are found in many shapes depending on the synthesis performed, but in biomedical applications the spherical form is desired in most cases. Their ability of not inducing cells apoptosis or cells damages is the reason why the spherical form is preferred in the experimental studies. Another important characteristic of silver nanoparticles is the optical activity. Silver, an alkali metal, is able to absorb in the visible domain of electromagnetic spectrum [4], so its use in biophysical studies have become an interesting research. The nanoscale level of silver particles offers them a strong antibacterial character. Able to perform this property on a high number of fungi and bacteria [6], silver nanoparticles are nowadays used in antimicrobial applications [7]. Based on the chemical reduction of $\mathrm{Ag}^{2+}$ ions, silver nanoparticles interfere with internal structure of bacteria and limits their microbial effect at minimum.

The importance to maintain a nanoscale dimension on silver nanoparticles led to the development of a non-conventional tracking analysis method. In order to detect the main factors involved in the early stage of synthesis and to track the nanoparticles trajectories in liquid suspensions, the biophysical method Direct Particle Tracking (DPT) can be adjusted for nanoparticles distribution studies. The dynamics of nano and micro-particles in biological systems strongly influence the interactions between particles [8]. Particle motions depending on the media properties is also influenced by the diffusion of particles in suspensions over time [9]. A real-time analysis technique of such nanoparticles in liquid media can even show the dynamic process in which nano and micro-particles interact with cells by scattering the diffused light using an optical system. Another important process that can be visualized using DPT is aggregation. Aggregation can occur fast [10], so an optical pathway, such it is used in DPT technique, can determine the possible clusters formed without any other chemical reagent added. Nanoparticle aggregation is an essential process in medical applications. The mobility of such particles in presence of biological structures must be determined in experimental setups in order to prove that an increase in particle dimension will not interfere with the diffusion process in cell or tissue membranes.

The variety of methods used to obtain nanoparticles depends on many factors and can interferes with the aggregation process. The chemical synthesis of nanoparticles presents the advantages of a low-cost and of the possibility to control the nanoparticles dimension depending on the reagent introduced in the synthesis. The co-precipitation is the most affordable method of synthesis for silver nanoparticles. The short time and the low-cost are encouraging factor in choosing this method. The co-precipitation, from a physical point of view, is based on an aqueous solution in which particles are in Brownian movement. After the fabrication process, nanoparticles can be observed and analyses through DPT technique, which is based on the dimensional analysis of particles found in a solution. This technique is useful in the early stage of synthesis process in order to observe if the experimental synthesis worth to be implemented in the laboratory. A virtual experiment sustained by mathematical simulations, which will be performed in this paper, can easily show a dimensional analysis of nanoparticle's distribution. Also, such simulations can prove the nanometric scale of the particles found in a solution. The results obtained through a simulated DPT process will reduce time or chemical reagents and will indicate the accuracy of this method which can be further improved and developed.

DPT technique is an optical procedure able to visualize in real time the dynamics and the trajectory of each nanoparticle. Based on an optical setup, the Brownian movement of each 
particle diffused is observed and analyzed by a software program [11]. The Brownian movement is captured using a system based on an optical substrate on which the sample is positioned. The substrate is composed by a crystal able to diffract the light with a specific refractive index to the sample. In the moment that a light beam, from a laser source, is incident on the suspension placed on the substrate, each particle from the media is able to capture and scatter light in a certain amount, as it can be observed on the right side of Fig. 1. The CCD camera placed on the microscope setup visualizes each individual particle on each frame. The video is analyzed by software programs able to determine the particle size distribution depending on a temporal component. The schematic form of the optic pathway can be observed on the left side of Fig. 1.

This optical technique is able to provide many types of data. First, it can produce the mean hydrodynamic size of each particle and second, it can also output the distribution of spherical nanoparticles in the solution comparing to other light scattering techniques [12].

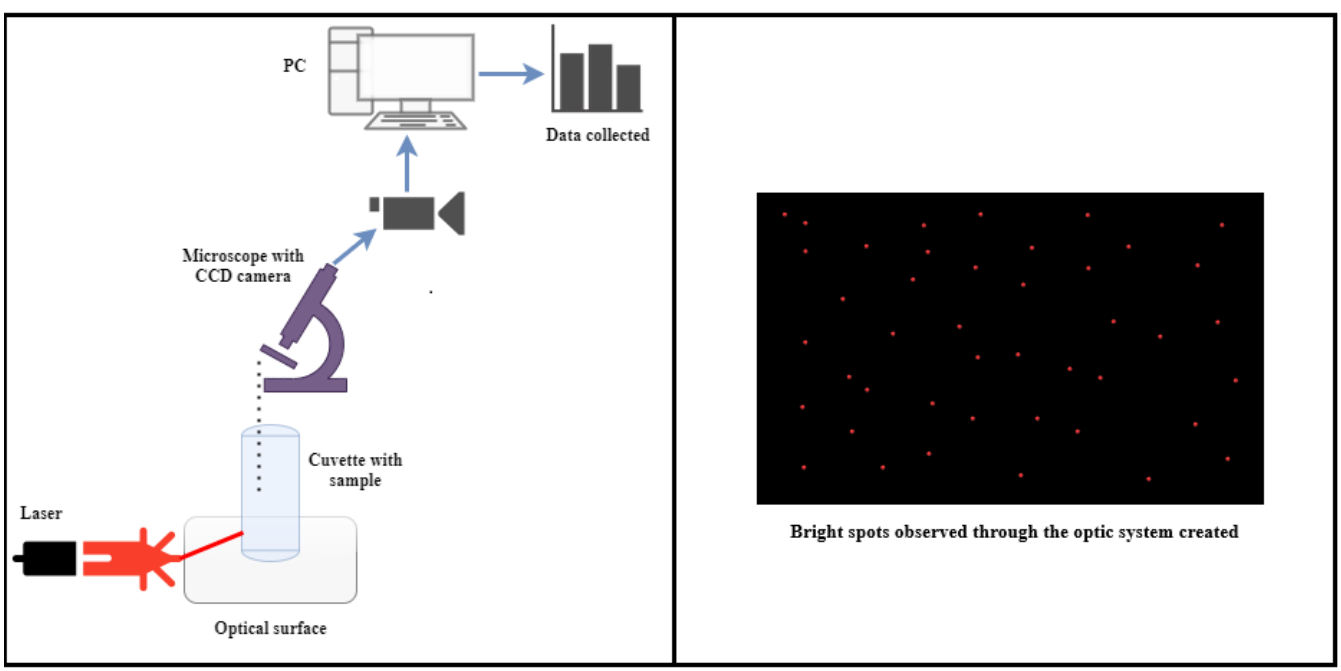

Fig. 1. Schematic pathway of DPT technique

DPT technique can determine the dispersity and the diameter of nanoparticles in samples or the degree of agglomeration that occurred. In order to possess a strong antibacterial effect, silver nanoparticles, for example, should present a small nanometer dimension, so this proportionality relationship must be carefully studied. The interaction with cells also depends on the stability and dimension of nanoparticles. The release of silver ions should be decreased in contact with tissues and should be able to intensify in a bacterial infected area. The ability of penetrating bacteria membrane is correlated with the (small) diameter of nanoparticles.

Mathematical simulations for DPT technique will determine the dispersity of these diffused nanoparticles in liquid suspensions by obtaining individual trajectory for particles and the specific diffusion time. Such information will be addressed in the synthesis step of experiments. The ability to distinguish between many types of nanoparticles based on their sizes [11], with and without any functionalization for example, is a very important benefit that this optical technique offers. The functionalization process is strongly practiced for silver or magnetite nanoparticles, so data about the change in diffusion time can be fundamental for the final application. 


\section{Direct particle tracking - theoretical considerations}

Over years, many studies based on technique such as Dynamic Light Scattering or Single Tracking Particle [8] were developed in order to prove the importance of particle size. Beside them, microscopy techniques have been used in order to characterize the solution from the sample. Microscopy techniques are able to provide valuable information about the morphology of particles and about their distribution in the sample, but they are suitable only in small area of investigation. Another disadvantage is based on the modifications that could appear in the sample. Such techniques require a careful preparation before analysis, so a possible aggregation appeared in the entire population could be disturbed during the preparation steps [13].

DPT is a biophysical method based on the Brownian diffusion of particles in the liquid medium from the setup cavity. According to many literature studies, this type of diffusion is explained by the random movement of particles in a fluid [14]. The ability of particle to possess such Brownian motion is due to the interactions appeared between particles in fluids. This molecular phenomenon, explained through many mathematical equations which will be further explained, provides a chaotic dynamic [15] in the movements of particles, as it can be observed in Fig. 2.

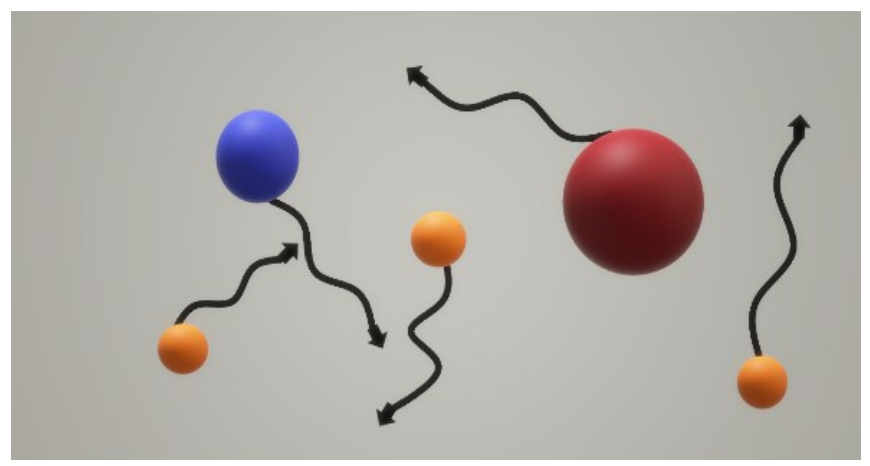

Fig. 2. Brownian diffusion of nanoparticles in the fluid device

The trajectories of random motions of particles are observed according to the simple diffusion model. The process is based on the ability of atoms to provide interactions between molecules. An important advantage in this model is represented by the low resolution in which particles can be visualized. At a nanoscale level, DPT offers valuable information about spatial characteristics of each nanosphere. Trajectories of nanoparticles can be different depending on the medium used. For example, it has been proved that biological solutions possess a certain impact on the diffusion of particles based on the atoms present in the liquid [8]. This experiment can be explained by the heterogeneous character of biological substances which obstruct a free movement of particles [16]. Beside the spatial component, the temporal characteristic is also involved in this process. Once the distance of a motion is decreased, the time component is also shorter [8], thus maintaining a relationship of direct proportionality between them.

The diffusion coefficient of particles is determined using a two-dimensional system and a mathematical analysis. Each trajectory is recorded in a $(x, y)$ system with a certain number of frames/second, so for every time in which a particle is found, a quantitative parameter called mean square displacement (MSD) can be calculated [9]:

$$
\begin{gathered}
M S D\left(\Delta t_{n}\right)=M S D_{x}(\Delta t)+\operatorname{MSD}_{y}(\Delta t) \\
M S D\left(\Delta t_{n}\right)=\frac{1}{N-1-n} \sum_{j=1}^{N-1-n}\left\{[x(j \delta t+n \delta t)-x(j \delta t)]^{2}+\left[y(j \delta t+n \delta t)-y(j \delta t)^{2}\right\}\right.
\end{gathered}
$$




$$
\Delta t_{n}=n \delta t
$$

where $x(j \delta t+n \delta t)$ and $y(j \delta t+n \delta t)$ correspond to the particle position in the 2-D system after the particle moved from the initial $(j \delta t)$ position with $\Delta \mathrm{t}_{\mathrm{n}}=n \delta t$ time. $\mathrm{N}$ correspond to the frames found in the video recorded, $n$ and $\mathrm{j}$ are positive integers, while $\mathrm{n}$ is represented by the time increment [9]. A value which could not be integrated in a specific range of dimension can be associated with an increase diffusion coefficient. This can appear when a super-diffusion, an accelerant motion of particle, is achieved in the sample [16]. An experimental study developed in 2015 by a group of researchers has shown that the diffusion coefficient for a single particle can be $0,27 \times 10^{-9} \mathrm{~cm}^{2} \mathrm{~s}^{-1}$, while for entrapped particles or multiple particles, the diffusion coefficient increases up to $1,3 \times 10^{-9} \mathrm{~cm}^{2} \mathrm{~s}^{-1}[16]$.

After the MSD is obtained, the diffusion coefficient can be analyses for each nanoparticle from the suspension. The simple diffusion of molecules is analyzed depending on the $\mathrm{x}$ and $\mathrm{y}$ directions of the system $[8,9]$ :

$$
\begin{gathered}
M S D_{x}(\Delta t)=2 D_{x} \Delta t ; M S D_{y}(\Delta t)=2 D_{y} \Delta t \\
4 D=2 D_{x}+2 D_{y} \\
M S D\left(\Delta t_{n}\right)=4 D \Delta t
\end{gathered}
$$

where $\mathrm{D}$ is the diffusion coefficient, $\mathrm{D}_{\mathrm{x}}$ is the diffusion coefficient on $\mathrm{x}$ axis and $\mathrm{D}_{\mathrm{y}}$ is the diffusion coefficient on y axis [9]. The difference of time between the initial one and the final time should be short in order to avoid the duplication of nanoparticles trajectories, but long enough so that analysis could be performed. After the mathematical formulas, the implementation of MSD in diffusion coefficient analysis led to a specific value of D parameter calculated for each nanoparticle or microparticle from the system studied.

As it was already mentioned, DPT technique is innovative by allowing a live monitoring of nanoparticles flow in the sample. The sample is maintained in a liquid state, so the media is similar to the biological structures from the skin. In this technique, after the determination of diffusion coefficient, each individual nanoparticle dimension is determined using the StokesEinstein equation $[12,17]$, described in many literature studies:

$$
D=\frac{k T}{3 d \pi \text { ? }}
$$

where $\mathrm{d}$ is the hydrodynamic diameter, $\mathrm{k}$ is the Boltzmann constant, $\mathrm{T}$ is the temperature, $\mathrm{D}$ is represented by the diffusion coefficient and $\eta$ is the viscosity of the medium used [12]. The properties of each medium used can influence the final results. The viscosity found in the suspension can decrease the diffusion process, so nanoparticles would have a lower motion. Another important aspect is related to the temperature in the sample. In order not to create thermal agitation, a constant temperature should be achieved. Chemical structure of nanoparticle is also a parameter which can disturb the diffusion in the sample. Ionic strengths or particle surface could increase the reactivity of ions, so faster diffusion could be obtained [18]. Even if classical techniques are also based on this equation, sample preparation and problems in multimodal dispersions analysis are disadvantages which could be overcome by DPT technique. The Stokes-Einstein equation allows the determination also of nanoparticles concentration [17], so a mathematical simulation in this case will considerably reduce the losses and the inconvenient which could appear in the experimental setups.

In nanoparticle tracking techniques, the velocity $(v)$ is another important factor which can modify the diffusion process. Velocity profile is usually analyzed through fluorescent tracers based on the average displacements of the particle [19]. Since chemical fluorescent could interfere with the analysis process, the use of individual particles as a tracer in the laminar 
flow of a fluid is a characteristic of DPT technique. According to a recent study described in literature, the standard deviation extracted from the Brownian movement $\left(v_{S D}\right)$ interferes with the velocity flow according to the relationship [19]:

$$
v_{S D}=\sqrt{\left(\frac{2 D}{\Delta t}\right)}
$$

The results of the study have proved that an accurate velocity value is achieved once the standard deviation of Brownian movement is determined for each nanoparticle considered tracer. The velocity distribution of the particles in the fluid is determined by identifying each nanoparticle as a bright spot which scatters light. From the $\mathrm{x}$ and $\mathrm{y}$ axis it is extracted the displacement distribution of the luminous spots, while the ambiguity of the Brownian movement for each particle must be eliminated for accurate results [19]. Based on the optical pathway described the flow in the sample can be determined, so its influence on the diffusion of each particle can be determined.

Mathematical simulations of Brownian motions and diffusion trajectories using software programs will show a prototype in particles dynamics. The ability to determine the optical diffusion coefficient and the expected dimensions will reduce significant the challenges appeared in the experimental model. The histograms obtained through mathematical simulations, based on diameter of each particle and on the diffusion process in time, will prove the small dimensions of particles and will be able to identify the possible clusters formed depending on the number of particles tracked. The synthesis of silver nanoparticles will be able to be considered a good choice and the most important factors will be observed through these theoretical simulated experiments.

\section{Brownian motion simulation}

The Brownian motion was simulated using the functions in CHODIN code [20-23], which can generate DLS time series, far interference field and a succession of equally time spaced frames of the interference field that are joined in a recording and simulate the boiling speckles far field.

The number and the physical parameters of scattering centers SCs in suspension, experimental setup geometry, data acquisition geometry and acquisition rates are input parameters. The code moves each SC individually for each simulation step. The velocity distribution was modeled as being a Maxwell - Boltzman distribution, which is the product of the three velocity distribution functions for one dimension:

$$
f_{v}\left(v_{i}\right)=\sqrt{\frac{m}{2 \pi k T}} \cdot \exp \left(\frac{-m v_{i}^{2}}{2 \pi k T}\right)
$$

In (9) $\mathrm{k}$ is Boltzman's constant, $\mathrm{T}$ is the absolute temperature, $\mathrm{m}$ is the mass of the particle in thermal equilibrium with the environment and $\mathrm{i}$ stands for either $\mathrm{x}, \mathrm{y}$ or $\mathrm{z}$. At each simulation time step the velocity values for $\mathrm{v}_{\mathrm{x}}, \mathrm{v}_{\mathrm{y}}$ and $\mathrm{v}_{\mathrm{z}}$ of each $\mathrm{SC}$ were generated using random numbers with a normal distribution having the variance $\mathrm{kT} / \mathrm{m}$. For particles that are diffusing in a solvent, the number density $\mathrm{n}(\mathbf{r}, \mathrm{t})$ variation in time is described by the diffusion equation:

$$
\frac{\partial n}{\partial t}-D \cdot \nabla^{2} n=0
$$

where $\mathrm{D}$ is the diffusion coefficient, described by Stokes-Einstein equation (7). The probability $\mathrm{P}(\mathbf{r}, \mathrm{t})$ of finding a particle in a small volume is directly proportional to the number density, this leads to the equation (11), where $\mathrm{D}$ is the diffusion coefficient, 


$$
\frac{\partial P}{\partial t}-D \cdot \nabla^{2} P=0
$$

A solution, for initial conditions where all the particles are in origin at $\mathrm{t}=0$ is:

$$
P(\vec{r}, t)=(4 \pi D t)^{\frac{-3}{2}} \cdot \exp \left(\frac{-r^{2}}{4 D t}\right)
$$

The solution of the diffusion equation, the probability distribution $\mathrm{P}(\mathbf{r}, \mathrm{t})$ can be connected with the second moment of the $\mathrm{P}$ distribution, $\left\langle\mathrm{r}^{2}\right\rangle$, which is measurable, and this leads to:

$$
\left\langle r^{2}(t)\right\rangle=\int_{0}^{\infty} \int_{0}^{\pi} \int_{0}^{2 \pi} r^{2} \cdot P(\vec{r}, \mathrm{t}) \cdot r^{2} \cdot \sin \theta \mathrm{d} \theta d \phi=6 D t
$$

A computer experiment, as described before, moving the SCs with the procedure that simulates the Brownian motion, was carried on and the final position of each particle was recorded. Further details on the CHODIN code can be found in [20-22] and [23].

\section{Results and discussions}

A computer simulation using the procedure described in the previous section was carried on for different number of particles, diameters and diffusion time. The experiment was carried on to shorten the experimental effort and to find a proper number of particles to be tracked and a proper diffusion time, and, of course, to verify that the procedure and the computer code written to analyze the diffusion information extracted from consecutive frames actually works well.

The diffusion is simulated in three dimensions by the CHODIN code, but only the diffusion in two dimensions is examined, as a recording in the microscope field is the projection of the motion in two dimensions. The results for a number $\mathrm{N}=100$ particles, having a diameter of $1.6^{*} 10^{-7} \mathrm{~m}$, were chosen to be presented. The solvent was pure water at $20^{\circ} \mathrm{C}$, with the dynamic viscosity coefficient of $10^{-3} \mathrm{daP}$. The particles were allowed to evolve and the positions were saved for each particle at each frame. The framerate was considered to be 10 per second and a total number of 200 frames. The particles were assumed to be magnetite $\left(\mathrm{Fe}_{3} \mathrm{O}_{4}\right)$ with a density of $5180 \mathrm{~kg} / \mathrm{m}^{3}$. Each particle was assumed to be at $(0,0,0)$ at the beginning of the simulation.

The trajectories of three particles are illustrated in Fig. 3. We notice the random motion of the three particles as time passed. This result shows the random distribution of particles in the solvent in a 2-D system, so the Brownian movement of each particle is observed and proved. The diffusion rate is different for each particle, this result suggesting a diversity of the particle's size and behavior in a liquid suspension.

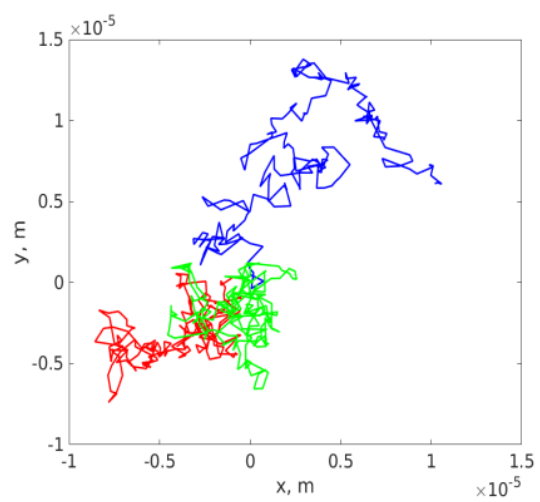

Fig. 3. Trajectories of three particles during simulated diffusion in XOY plane. 
The recorded data was analyzed for each particle and each frame, calculating the displacement with eq. (4) for each consecutive sequence of frames, the mean squared displacement for each particle and the diffusion coefficient with eq. (6), followed by the diameter of the particle using eq. (7). The histogram of the diameters for the simulated diffusion experiment are illustrated in Fig. 4.

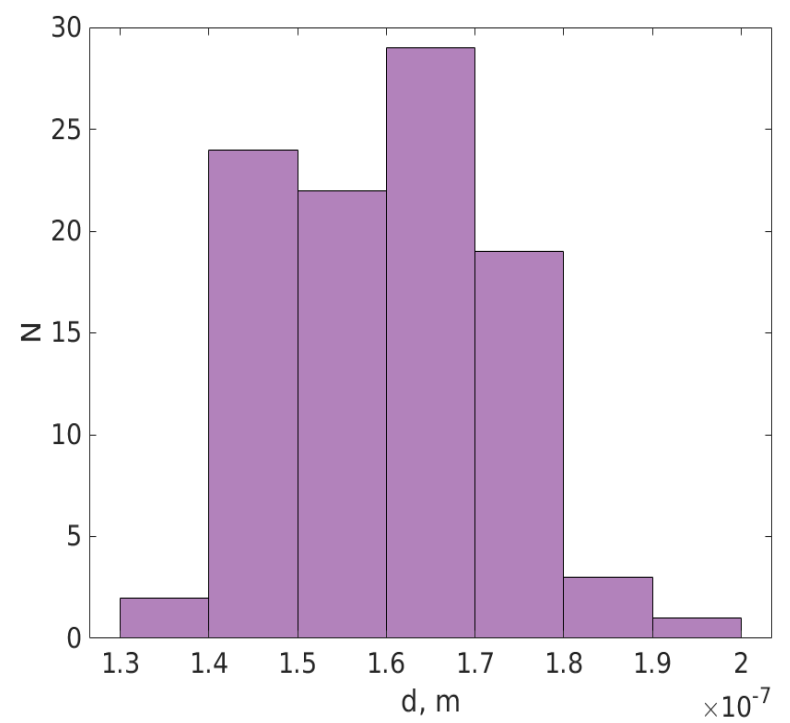

Fig. 4. The histogram of the computed diameters

Examining Fig. 4 we notice that most of the diameters were found to be in the range $1.4-$ $1.8^{*} 10^{-7} \mathrm{~m}$, which is consistent with the value of $1.6^{*} 10^{-7} \mathrm{~m}$ that was used for simulation. The Gaussian shape of the histogram obtained in the graph confirms the diversity of particle's diffusion rate observed in the first result presented in Fig. 3. The results of mathematical simulations strongly suggest a nanometer dimension for the particle investigated, so the method is promising to be investigated in an experimental setup.

The spread is caused by the small number of particles, which was 100 and by the overall small number of simulation steps and by the essence of the simulation, which is a Monte Carlo type. The accuracy can be improved by tracking a bigger number of particles for a bigger time interval.

\section{Conclusions}

The results of the computer simulation presented here proved both that the DPT method is valid and that the software written for DPT data analysis works well. The results shown a nanometer dimension for the particles studied, so further experimental setups will be accomplished. As compared with the DLS procedure [24-26], DPT is slower and requires more experimental and computational effort, especially when we compare it with the novel Artificial Neural Network assisted DLS procedure [27-29], but presents the advantage of providing the particle size distribution, instead of the average size. Another advantage of DPT as compared with the DLS is that it is not affected by the fact that bigger particles scatter light more efficient than smaller particles [30 -32], therefore the average provided by DLS is affected by systematic errors, while an average computed from the DPT result is, therefore, more accurate.

DPT technique, which is based on a dimensional analysis in suspensions or nanoparticles solutions, allows the study of particles found in a continues Brownian movement in solutions. 
Such information obtained from this innovative technique determine the specific distribution of particles, an important aspect in case of biomedical applications. Mathematical simulations performed before the experimental part validates the correctness and show the reproducibility of the method. The results obtained in this paper shown that DPT technique can offer important data about the nanoparticle's dimension and distribution in a solution. So, by performing these simulations, we proved that the method is able to be performed on real data in the early stage of silver nanoparticles synthesis.

\section{References}

1. A. Mecke, I. Lee, J.R. Baker jr., M.M. Banaszak Holl, B.G. Orr, Eur. Phys. J. E 14, 7 (2004)

2. J. Jeevanandam, A. Barhoum, Y.S. Chan, A. Dufresne, M.K. Danquah, Beilstein journal of nanotechnology, 9, 1050-74 (2018)

3. S. Sahani, Y.C. Sharma, Food Chemistry, 342, 128318 (2020)

4. I. Khan, K. Saeed, I. Khan, Arabian journal of chemistry, 12, 908-31 (2019)

5. B. Khodashenas, H.R. Ghorbani, Arabian Journal of Chemistry, 12, 1823-38 (2019)

6. J. Deng, Q.M. Ding, M.X. Jia, W. Li, Z. Zuberi, J.H. Wang, et al, Environmental Pollution, 275, $116662(2021)$

7. T. Naseem, T. Durrani, Environmental Chemistry and Ecotoxicology, 3, 59-75 (2021)

8. H. Qian, M.P. Sheetz, E.L. Elson, Biophysical journal, 60, 910-21 (1991)

9. A. Kusumi, Y. Sako, M. Yamamoto, Biophysical journal, 65, 2021-40 (1993)

10. P. Babakhani, Scientific Reports, 9, 14071 (2019)

11. https://www.azonano.com/article.aspx?ArticleID=2488, [Accessed at 16.03.2021]

12. B.M. Hoover, R.M. Murphy, Journal of pharmaceutical sciences, 109, 452-63 (2020)

13. H. Lee, D.-B. Kwak, S.C. Kim, D.Y. Pui, Powder Technology, 355, 18-25 (2019)

14. X. Bian, C. Kim, G.E. Karniadakis, Soft Matter, 12, 6331-46 (2016)

15. E.E. Michaelides, Journal of Fluids Engineering, 138, 051303 (2016)

16. H. Al-Obaidi, A.T. Florence, Journal of Drug Delivery Science and Technology, 30, 266-77 (2015)

17. K.P. McComiskey, L. Tajber, European Journal of Pharmaceutics and Biopharmaceutics, 130, 314-26 (2018)

18. M. Mahdavi, M. Sharifpur, M.H. Ahmadi, J.P. Meyer, Journal of Thermal Analysis and Calorimetry, 135, 111-21 (2019)

19. Y. Matsuura, A. Nakamura, H. Kato, Sensors and Actuators B: Chemical, 256, 1078-85 (2018)

20. D. Chicea, Applied Optics, 47, 1434-42 (2008)

21. D. Chicea, ROMOPTO 2009, Using CHODIN to simulate the dynamics of coherent light scattering on nanofluids, (Sibiu, Romania, 2010)

22. D. Chicea, INDLAS 2007, CHODIN-a computer code to simulate coherent light scattering dynamics on biological suspensions, (Bran, Romania, 2007)

23. D. Chicea, Romanian Journal of Physics, 51, 403-9 (2004)

24. D. Chicea, Current Nanoscience, 8, 259-65 (2012)

25. D. Chicea, Optoelectron Adv Mater Rapid Commun, 3, 1299-305 (2009)

26. D. Chicea, E. Indrea, C. Cretu. Journal of optoelectronics and advanced materials, 14, 460-6 (2012)

27. D. Chicea, Measurement Science and Technology, 28, 055206 (2017)

28. D. Chicea, S.M. Rei, Measurement Science and Technology, 29, 105201 (2018)

29. D. Chicea, Sensors, 20, 3425 (2020)

30. D. Chicea, I. Turcu, Journal of Optoelectronics and Advanced Materials, 8, 1516 (2006)

31. M. Răcuciu, D. Creangă, A. Airinei, D. Chicea, V. Bădescu, Materials Science-Poland, 28, 609-16 (2010)

32. D. Chicea, I. Turcu, Optik, 118, 232-6 (2007) 